\title{
Long-term Efficacy and Safety of Cardioneuroablation in Patients with Vagally Mediated Bradyarrhythmias
}

\author{
(1) Tümer Erdem Güler, () Tolga Aksu
}

Kocaeli Derince Training and Research Hospital, Clinic of Cardiology, Kocaeli, Turkey

\begin{abstract}
Objectives: Long-term efficacy and safety are still uncertain in patients with vagally mediated bradyarrhythmias (VMB) undergoing cardioneuroablation (CNA). Thus, we assessed the outcome of CNA in those patients during long-term follow-up using clinical history in outpatient visits.

Materials and Methods: A total of 27 patients (42.7 \pm 14 years; $17(63.0 \%$ men $)$ were included in this study. The main mechanism involved in the occurrence of syncope was vasovagal syncope in $16(59.3 \%)$ patients, functional atrioventricular block (AVB) in seven (25.9\%) patients, and sinus bradycardia or pauses in four (14.8\%) patients. The ablation strategy included right-sided or bi-atrial ablation of ganglionated plexi. Syncope recurrences were assessed clinically during follow-up.
\end{abstract}

Results: Acute procedural endpoints were achieved in 26 (96.2\%) of cases. During a median follow-up period of 52 months (IQR, 28-56 months), all but 2 (7.4\%) of 27 patients were free of new syncope. Significant clinical and ECG improvement was detected in four (57.1\%) of seven patients with AVB. In the remaining two patients except one patient with acute failure, pacemaker was implanted despite no syncope recurrence because evening time variable-degree AVB episodes were seen on follow-up Holter recordings. Procedure related complication was not seen in any cases. Symptoms attributed inappropriate sinus tachycardia was observed in two patients.

Conclusion: Our results demonstrate long-term efficacy and safety of cardiovascular autonomic neuropathy in patients with VMB. Randomized controlled studies are needed to define long-term efficacy and safety of cardioneuroablation.

Keywords: Vasovagal syncope, atrioventricular block, sinus node dysfunction, bradycardia, ganglionated plexi

Address for Correspondence: Tolga Aksu, Kocaeli Derince Training and Research Hospital, Clinic of Cardiology, Kocaeli, Turkey e-mail: aksutolga@gmail.com ORCID: orcid.org/0000-0001-8061-9660

Received: 17.02.2020 Accepted: 24.02.2020

Cite this article as: Güler TE, Aksu T. Long-term Efficacy and Safety of Cardioneuroablation in Patients with Vagally Mediated Bradyarrhythmias. EJCM 2020;8(1):28-34.

DOI: 10.32596/ejcm.galenos.2020.02.07

${ }^{\circ}$ Copyright 2020 by Heart and Health Foundation of Turkey (TÜSAV) / E Journal of Cardiovascular Medicine published by Galenos Publishing House. 


\section{Introduction}

Parasympathetic excessive-activity or an imbalance in control of sympathetic and parasympathetic systems may be the actual reason of some clinical conditions such as sinus bradycardia or pauses (SND), vasovagal syncope (VVS), and vagal atrioventricular block (AVB) (1). Vagally mediated bradyarrhythmias (VMB) might be used to define these different clinical conditions ${ }^{(2)}$. Great majority of those cases might be very symptomatic and resistant to non-invasive therapies and medications. Ganglionated plexi (GPs) contain postganglionic neuronal cells of parasympathetic system of the sinoatrial and atrioventricular (AV) nodes and tend to cluster in discrete epicardial or sub-epicardial sites ${ }^{(3,4)}$. Endocardial radiofrequency catheter ablation of these GPs is named as cardioneuroablation (CNA) and might be used to regulate deleterious effects of the autonomic processes occurring in $\mathrm{VMB}^{(5)}$.

The current study reports the long-term efficacy and safety of CNA in patients with VMB.

\section{Materials and Methods}

The study was approved by the Kocaeli Derince ethical committee (approval no: 2019-75). Informed consent of the individuals was waived because of the retrospective nature of the study.

\section{Design of Study}

This is a retrospective study in which patients having at least one syncope episode underwent CNA due to following primary diagnoses before enrollment: VVS; SND; and AVB. Some patients had more than one of these diseases at the same time. All these patients were thoroughly evaluated by history, Holter recording, headup tilt-test (HUT), exercise test, and atropine challenge test permitting to define the main cause of syncope. The main cause of syncope, number of syncopal episodes, and accompanying functional bradyarrhythmia diagnosis were recorded separately for all cases. Non-interventional therapies including optimal salt and fluid intake and physical counterpressure maneuvers were attempted in all cases before the procedure. Only patients refusing pacemaker implantation were enrolled.

\section{Atropine Challenge Test}

A $0.04 \mathrm{mg} / \mathrm{kg}$ intravenous atropine sulfate infusion was applied after 4-hour fasting period at least 24 hours before the procedure. ECG recordings were taken every two minutes during $30 \mathrm{~min}$. In case of VVS or SND, a positive atropine response was defined as R-R interval increased by $25 \%$ or $\geq 90 \mathrm{bpm}^{(5)}$. In patients with AVB, a decrease of $\geq 25 \%$ in PR interval was accepted as positive response. Achieved final sinus rate and PR interval were used to define acute autonomic denervation.

\section{Patient Selection (Table 1)}

In our previous works, patient selection criteria were discussed in detail ${ }^{(6,7)}$.

\section{Vasovagal Syncope}

All patients had at least 3 pre-enrollment syncopal episodes. In all patients, type $2 \mathrm{~B}$ or type 1 response with asystole longer than 3 seconds was confirmed by using $\operatorname{HUT}^{(8)}$.

\section{Atrioventricular Block}

The diagnosis of AVB refers to functional second or third degree AVB during 24-hour Holter recording. Only patients having at least one syncope episode were included in the study.

\section{Sinus Node Dysfunction}

Sinus asystole was defined as transient breakdown of atrial activity resulting in pauses longer than 2 seconds during 24-hour Holter recording. Only patients having at least one syncope episode were included in the study.

\section{Ganglionated Plexi Mapping and Ablation}

The procedures were conducted under conscious sedation. Based on a specific time point $\left(7^{\text {th }}\right.$ of December 2015), following approaches were used to define localization of GPs: (1) combination of high frequency stimulation (HFS) and spectral analysis and (2) electrogram (EGM) based strategy. 


\section{Combination Strategy}

In our previous strategy (before $7^{\text {th }}$ of December 2015), GPs were detected by using a combination of fast Fourier transform analysis, HFS and empirical anatomic ablation $^{(7)}$. Anatomical shell of the left atrium (LA) and the right atrium (RA) was created by using EnSite Velocity system (Abbott, Sylmar, CA, USA). In the patients with SND and VVS, left and right atrial parts of ganglion A and B were targeted and ablated, respectively (Figure 1). In patients with $A V B$, right-sided approach was used to ablate ganglion $\mathrm{A}$ and $\mathrm{C}$.

To define the localization of GPs, conventional EGMs were converted into the frequency domain potentials by using computer based offline analysis. The potentials

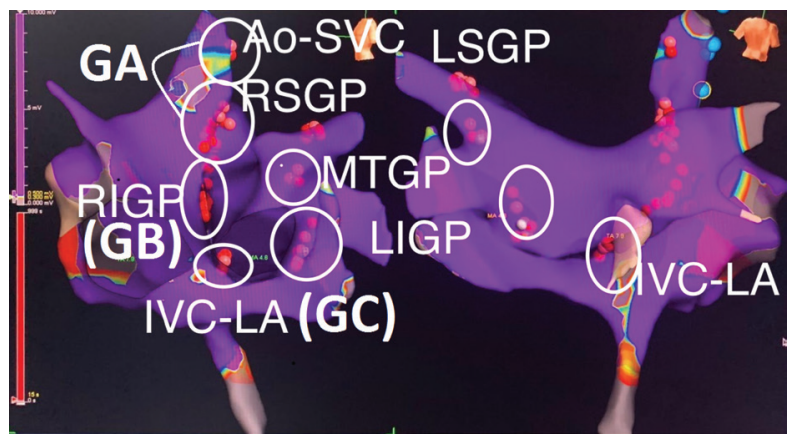

Figure 1. Schematic representation of ganglionated plexi. There are a lot of different classification systems to define the localization of ganglionated plexi (GPs). According to animal studies, there are 3 main GP sites which are located between left and right atrial structures. Ganglion A is accepted as a head station for parasympathetic innervations of sinus node, atrioventricular node, and both atria. Great majority of parasympathetic efferent fibers converge in ganglion A and project to other GPs. Although there is no clear definition for this anatomic area, according to the anatomical specimens both the aorta-superior vena cava GP (AoSVC GP) and the right superior/anterior GP (RSGP) constitute ganglion A. The Ao-SVC GP and the RSGP are located between the medial SVC and aortic root and between the SVC and the right superior pulmonary vein (RSPV), respectively. Ganglion B (GB) is located between the right pulmonary veins and the right atrium in interatrial septum area. The right inferior/posterior (RIGP) may also be used to define this GP. Ganglion C (GC) or the inferior vena cava-left atrium GP (IVC-LA GP) is located between the posterior wall of the LA and IVC around coronary sinus (CS) ostium. The left superior GP (LSGP) is located on the roof of the left atrium, between left superior PV (LSPV) and the left atrial appendage. The Marshall tract GP is located anterior to the left inferior PV (LIPV). The left inferior GP (LIGP) is identified on the posterior lateral surface of the left atrial base on the atrial side of the atrioventricular groove demonstrating fractionated pattern were tagged in the 3D map. All fractionated sites were checked for positive vagal response (second- or third-degree AVB or or R-R interval increased by $50 \%$ ) by using HFS. The sites demonstrating positive vagal response were targeted as GP. The upper limits of power and temperature were set to $35 \mathrm{~W}$ and $43{ }^{\circ} \mathrm{C}$ with a cooling rate of $18 \mathrm{~mL} / \mathrm{min}$, respectively. After each radiofrequency application, response to HFS was checked at the same site. In the existence of continuing positive response, ablation line was extended to the adjacent site to form a cloudlike shape until completely elimination of vagal response.

\section{Electrogram Based Strategy}

Because we realized a significant correlation between fractionated potentials in fast Fourier transform analysis and fragmented EGMs in conventional electrophysiological recordings, we decided to use fragmentation pattern of EGMs to detect localization of GPs after the $7^{\text {th }}$ of December $2015^{(8)}$. After the creation of the 3D map of both atria, bipolar endocardial atrial EGMs were evaluated for number of deflections at filter settings of 200-500 Hz. The EGMs demonstrating more than four deflections in a region that was consistent with probable localization of GPs were targeted for ablation in both atria (Figure 1,2). In addition to ablation of ganglion $\mathrm{A}$ and $\mathrm{B}$, the left superior and the left inferior GPs were ablated in the existence of fragmented EGMs (Figure 1,2). If procedural endpoints were not achieved, anatomic ablation was conducted around ganglion A, B, and $\mathrm{C}$ sites. Radiofrequency energy was limited to 35 watts (W) along the roof under an irrigation flow rate of $17 \mathrm{~mL} / \mathrm{min}$, and $40 \mathrm{~W}$ in the remaining areas.

\section{Endpoints}

Ablation endpoints: (1) elimination of targeted EGMs; and (2) inability to induce a positive vagal response with repeat HFS or radiofrequency application.

Clinical endpoint was inability to cause positive response with repeat atropine challenge.

Acute success: achievement of both ablation and clinical endpoints. 
Table 1. Detailed inclusion and exclusion criteria in patients with vasovagal syncope, functional atrioventricular block, and sinus node dysfunction

\begin{tabular}{|c|c|c|c|}
\hline & & Inclusion criteria & Exclusion criteria \\
\hline \multirow[t]{4}{*}{ VVS } & \multirow[t]{4}{*}{ History } & $\begin{array}{l}\text { At least } 3 \text { vasovagal syncope episodes in the } \\
\text { preceding } 12 \text { months }\end{array}$ & $<3$ syncope episodes \\
\hline & & $\begin{array}{l}\text { VASIS type } 2 \mathrm{~B} \text { response or type } 1 \text { (mixed) response with } \\
\text { asystole of greater than } 3 \mathrm{~s} \text { on Head-up tilt table test }{ }^{*}\end{array}$ & $\begin{array}{l}\text { VASIS type } 2 A \text { and VASIS type } 3 \text { response on } \\
\text { Head-up tilt table test }\end{array}$ \\
\hline & & Positive response to atropine test & Negative response to atropine test \\
\hline & & 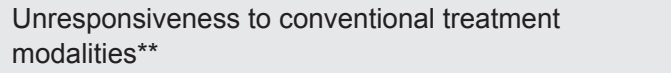 & \\
\hline \multirow[t]{8}{*}{ AVB } & EPS & Supra-Hisian AVB & Intra/infra-Hisian AVB \\
\hline & \multirow{4}{*}{ Paroxysmal AVB } & No AVB on baseline ECG & AVB on baseline $\mathrm{ECG}^{* * *}$ \\
\hline & & PR prolongation before AVB & Constant PR before AVB \\
\hline & & Decrease in sinus rate just before AVB & Increase in sinus rate just before AVB \\
\hline & & Resolving of $A V B$ with an increase in sinus rate & Resolving of AVB with a constant sinus rate \\
\hline & \multirow[t]{3}{*}{ Persistent AVB } & $\begin{array}{l}\text { AVB or intraventricular conduction disease on } \\
\text { baseline ECG }\end{array}$ & Unresponsiveness to atropine infusion \\
\hline & & $\begin{array}{l}\text { Complete resolution of AVB or conversion to first } \\
\text { degree AVB with atropine infusion }\end{array}$ & Unresponsiveness to exercise test \\
\hline & & $\begin{array}{l}\text { Complete resolution of AVB or conversion to first } \\
\text { degree AVB with exercise test }\end{array}$ & Junctional rhythm after atropine infusion \\
\hline \multirow[t]{6}{*}{ SND } & \multirow[t]{2}{*}{ Initial evaluation } & & Structural heart disease \\
\hline & & & Drug-induced sinus bradycardia \\
\hline & \multirow[t]{2}{*}{ Holter } & Pause $>2$ seconds & No sinus pause \\
\hline & & Symptomatic daytime sinus bradycardia or arrest & $\begin{array}{l}\text { Correlation between symptoms and ECG is not } \\
\text { established }\end{array}$ \\
\hline & Atropine response & Positive & Negative \\
\hline & Electrophysiological study & $\begin{array}{l}\text { Atropine responsive abnormal baseline sinus node } \\
\text { function? }\end{array}$ & $\begin{array}{l}\text { Atropine unresponsive abnormal baseline sinus } \\
\text { node function }\end{array}$ \\
\hline \multicolumn{4}{|c|}{ 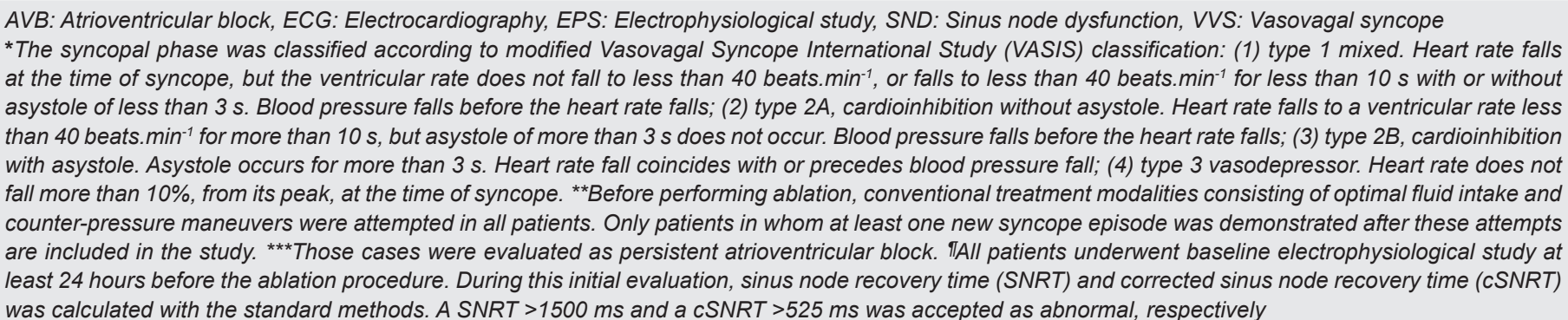 } \\
\hline
\end{tabular}

Atropine test was repeated 30 minutes after CNA for acute autonomic evaluation. Continuation of positive vagal response was defined as acute procedural failure.

\section{Follow-up}

All the cases were asked to record any prodromal symptoms, palpitation and/or syncope episodes. Followup consisted of a clinical visit or contact by telephone every 12 months. ECG, Holter or HUT recordings were reexamined in case of new syncope episode. The existence of recurrent syncope and/or undergoing new pacemaker implantation was accepted as the primary study outcome. Prodromal symptoms were excluded in the final analysis.

\section{Statistical Analysis}

Statistical analysis was performed using SPSS software, version 22 (IBM Corp., Armonk, NY, USA). Continuous variables were reported as means \pm standard deviations or 
medians (interquartile range (IQR). Categorical variables were expressed as frequencies and percentages. The Kaplan-Meier method was used to estimate the primary study outcome over time.

\section{Results}

We enrolled 27 consecutive patients $(42.7 \pm 14$ years; 17 (63.0\% men). All patients completed follow-up. Details of patients' demographics are summarized in Table 2. The essential cause of syncope was reflex syncope in 16 (59.3\%) patients, vagal AVB in seven $(25.9 \%)$ patients, and SND in four (14.8\%) patients, respectively. The

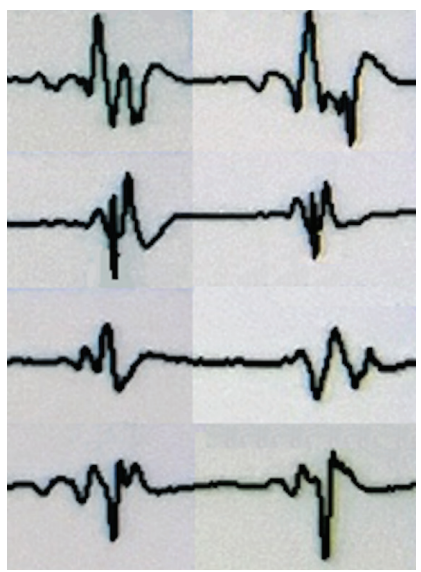

Figure 2. Schematic representation of fragmented intracardiac electrograms

\section{Survival Function}

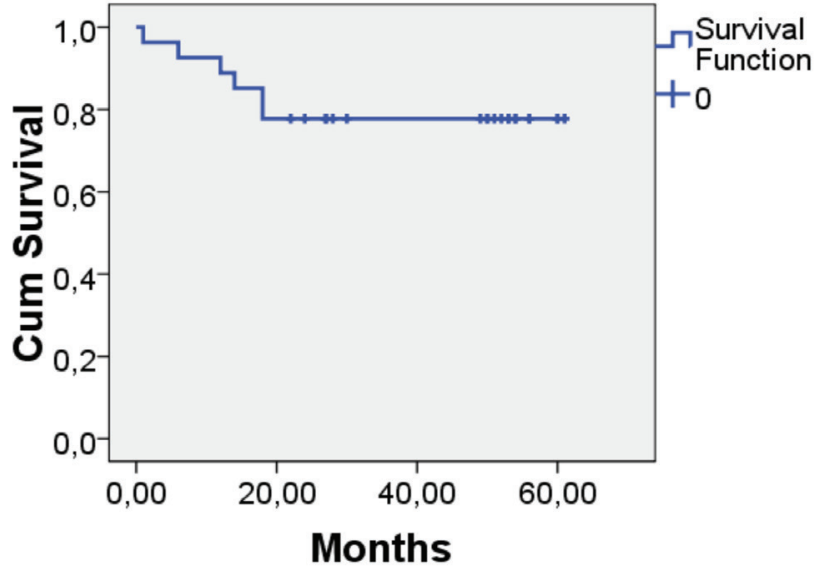

Figure 3. The Kaplan-Meier survival curve without primary endpoint in whole group

Cum: Cumulative types of conditions are displayed in Table 2. Combination strategy was attempted before the $7^{\text {th }}$ of December 2015 in $19(70.3 \%)$ of 27 cases. Acute success was achieved in 26 (96.2\%) of 27 cases. The median follow-up period was 52 months (IQR, 28-56 months).

\section{Vasovagal Syncope}

Syncope free survival was detected in $14(87.5 \%)$ of 16 patients with VVS. Two cases were admitted with new syncope episode at the $18^{\text {th }}$ and $14^{\text {th }}$ months after ablation. Repeated HUTs demonstrated type 3 (pure vasodepressor) responses in both patients. Oral midodrine hydrochloride was prescribed in these cases. At the end of post-medication 12-month follow-up period, patients were free from new syncope episode. HUTs were detected negative under treatment of midodrine. One VVS patient had accompanying daytime AVB before ablation. The patient demonstrated a significant clinical improvement with no syncope recurrence after CNA. However, pacemaker was implanted 6 months after CNA because follow-up Holter recordings demonstrated transient high-degree AVB at night.

Table 2. Baseline patient characteristics

\begin{tabular}{|c|c|c|}
\hline \multirow{6}{*}{ Baseline characteristics } & \multicolumn{2}{|l|}{ All patients $(n=27)$} \\
\hline & Age (years) & $42.7 \pm 14$ \\
\hline & Gender (male) (n/\%) & $17(63.0 \%)$ \\
\hline & $\begin{array}{l}\text { Pre-enrollment } \\
\text { syncope burden (n) }\end{array}$ & $4.2 \pm 2$ \\
\hline & $\begin{array}{l}\text { Pre-enrollment } \\
\text { medication use }(\mathrm{n} / \%)^{*}\end{array}$ & $17(62.9 \%)$ \\
\hline & Follow-up (months) & $46.0 \pm 13$ \\
\hline \multirow{3}{*}{ Cause of syncope } & VVS & 16 \\
\hline & AVB & 7 \\
\hline & SND & 4 \\
\hline \multirow{6}{*}{$\begin{array}{l}\text { Type/types of } \\
\text { vagally mediated } \\
\text { bradyarrhythmia }\end{array}$} & VVS & 11 \\
\hline & AVB & 5 \\
\hline & SND & 4 \\
\hline & $V V S+A V B$ & 2 \\
\hline & SND+AVB & 2 \\
\hline & VVS+SND & 3 \\
\hline
\end{tabular}

VVS: Vasovagal syncope, AVB: Atrioventricular block, SND: Sinus bradycardia or pauses, $n$ : Number

${ }^{*}$ Drugs which alter autonomic nervous system function were included in the analysis 


\section{Atrioventricular Block}

A significant improvement in clinical and ECG parameters were detected in four $(57.1 \%)$ of seven patients with AVB during follow-up. The remaining two patients except one patient with acute failure, although indicating a significant clinical improvement with no syncope recurrences, underwent pacemaker implantation due to transient high-degree AVB at night and accelerated junctional rhythm-based complaints on Holter recordings, respectively.

\section{Sinus Node Dysfunction}

All four patients with pure SND demonstrated an increase in minimum and mean sinus rates and abolishment of Holter-detected sinus pauses. Bradyarrhythmia related symptoms were not seen during follow-up. Remaining nine patients demonstrating accompanying SND were free from new syncope during follow-up. Primary study outcome was observed in six $(22.2 \%)$ of 27 patients (Figure 3).

\section{Safety}

Procedure related complication was not seen in any cases. Two patients were admitted to outpatient clinic due to complaints related to inappropriate sinus tachycardia. In one of them, symptoms gradually decreased during followup period. Although the other case was asymptomatic during the first 12 months, she suffered from EHRA class 2 symptoms. The symptoms were completely resolved under the treatment of ivabradine.

\section{Discussion}

The main findings of this study are the following: (1) CNA by combined approach or by electroanatomicalmapping-guided strategy could effectively prevent recurrent spontaneous syncopal episodes in patients suffering from VMB; (2) the effect on vagal tone, persisted at least 24 months after CNA, as shown by durable clinical improvement; and (3) despite being an invasive method, no CNA related major complication was seen in any cases.

The present study is the first one evaluating long-term efficacy and safety of CNA in patients who underwent
CNA with different indications. Theoretically, CNA may change relative balance of the autonomic nervous system which demonstrates a correlation with various physiologic functions of the heart. Although there are still controversies about exact etiopathogenesis of VVS, an increased parasympathetic tone together with an attenuated sympathetic tone may contribute to cardioinhibitory and vasodepressor responses, respectively ${ }^{(1)}$. In patients with AVB or SND, fibrosis of atrioventricular conduction system or the sinus node is the most common cause of bradyarrhythmia. However, potential contribution of excessive vagal tone should be kept in mind in intermittent and event some permanent forms ${ }^{(1)}$. Previous studies related to potential effects of CNA broadly focused on VVS cases. In 2011, Pachon et al. ${ }^{(9)}$ firstly studied longterm clinical efficacy of CNA in patients with VVS. By using left and right atrial ablation, they detected syncope free survival in 40 (93.1\%) of 43 patients in a mean followup of $45.1 \pm 22$ months. Similar results were recently demonstrated by $\mathrm{Hu}$ et al. ${ }^{(10)}$ with left-sided strategy. During follow-up of 21.4 \pm 13.1 months, 106 (92.2\%) of 115 patients had no syncope recurrence. A similar success rate was repeated in the present study. No spontaneous or HUT-induced asystole was seen in follow-up. A new provoked HUT showed a vasodepressor response in three cases. It might be speculated that CNA may completely prevent a systolic based new syncope episode or may cause a change from Type 2B to Type 3 response in case of new syncope episode.

Long-term efficacy of CNA in SND and AVB has been studied less than $\operatorname{VVS}^{(5,7,11,12)}$. In a recently published trial, Debruyne et al. ${ }^{(12)}$ studied the potential role of restricted right atrial approach in patients with SND and VVS and demonstrated a similar efficacy. However, short-term effects were presented in this study. In the current work, right-sided approach was used in five (18.5\%) of 27 cases. There is no well-designed study investigating long-term efficacy of CNA. In a previously published study, pacemaker was implanted in four (44\%) of nine cases due to symptomatic AVB recurrence after $\mathrm{CNA}^{(13)}$. In the current work, longterm clinical success was detected in $5(55.5 \%)$ of nine cases with AVB. Continuation of Holter detected AVB episodes 
despite a symptomatic improvement was observed only in the patients with AVB in the present cohort. Although the small sample size prevents establishing a definite correlation, the most plausible explanation of this high non-responder rate may be the difficulty in the exclusion of structural component in AVB cases.

\section{Study Limitations}

This is a single-center study with a relatively small number of cases. To overcome potential limitations related to retrospective design, strict inclusion and exclusion criteria were used to select study patients. As another limitation, accompanying conditions were detected in seven $(25.9 \%)$ of 27 cases. Although the most possible diagnoses for the occurrence of syncope were tried to be defined by using different modalities, the pathophysiology of syncope may not be clear in all patients with accompanying SND or AVB.

\section{Conclusion}

Endocardial ablation of GPs appears as an effective and safe strategy with favorable long-term results in well selected patients with VMB. Further randomized, controlled and multicenter studies should be performed to confirm the promising results presented in this study.

\section{Ethics}

Ethics Committee Approval: The study was approved by the Kocaeli Derince ethical committee (approval no: 2019-75).

Informed Consent: Informed consent of the individuals was waived because of the retrospective nature of the study.

Peer-review: Externally peer-reviewed.

\section{Authorship Contributions}

Surgical and Medical Practices: T.E.G., T.A., Concept: T.E.G., T.A., Design: T.E.G., T.A., Data Collection or Processing: T.E.G., T.A., Analysis or Interpretation: T.E.G., T.A., Literature Search: T.E.G., T.A., Writing: T.E.G., T.A.
Conflict of Interest: No conflict of interest was declared by the authors.

Financial Disclosure: The authors declared that this study received no financial support.

\section{References}

1. Aksu T, Guler TE, Yalin K, Mutluer FO, Ozcan KS, Calò L. Catheter Ablation of Bradyarrhythmia: From the Beginning to the Future. Am J Med Sci 2018;355:252-65.

2. Aksu T, Güler TE, Mutluer FO, Oto MA. Vagal denervation in atrial fibrillation ablation: A comprehensive review. Anatol J Cardiol 2017;18:142-8.

3. Hanna P, Rajendran PS, Ajijola OA, et al. Cardiac neuroanatomy - Imaging nerves to define functional control. Auton Neurosci 2017;207:48-58.

4. Chiou CW, Eble JN, Zipes DP. Efferent vagal innervation of the canine atria and sinus and atrioventricular nodes. The third fat pad. Circulation 1997;95:2573-84.

5. Pachon JC, Pachon EI, Pachon JC, et al. "Cardioneuroablation"-New treatment for neurocardiogenic syncope, functional AV block and sinus dysfunction using catheter RF-ablation. Europace 2005;7:1-13.

6. AksuT, GolcukE, Yalin K, GulerTE, Erden I. Simplified Cardioneuroablation in the Treatment of Reflex Syncope, Functional AV Block, and Sinus Node Dysfunction. Pacing Clin Electrophysiol 2016;39:42-53.

7. Aksu T, Guler TE, Mutluer FO, Bozyel S, Golcuk SE, Yalin K. Electroanatomic-mapping-guided cardioneuroablation versus combined approach for vasovagal syncope: a cross-sectional observational study. J Interv Card Electrophysiol 2019;54:177-88

8. Brignole M, Menozzi C, Del Rosso A, et al. New classification of haemodynamics of vasovagal syncope: beyond the VASIS classification. Analysis of the presyncopal phase of the tilt test without and with nitroglycerin challenge. Vasovagal Syncope International Study. Europace 2000;2:66-76.

9. Pachon JC, Pachon EI, Pachon MZC, Lobo TJ, Pachon JC, Santillana TG Catheter ablation of severe neurally meditated reflex (neurocardiogenic or vasovagal) syncope: Cardioneuroablation long-term results. Europace 2011;13:1231-42.

10. Hu F, Zheng L, Liang E, et al. Right anterior ganglionated plexus: The primary target of cardioneuroablation? Heart Rhythm 2019;16:1545-51.

11. Zhao L, Jiang W, Zhou L, et al. Atrial autonomic denervation for the treatment of long-standing symptomatic sinus bradycardia in non-elderly patients. J Interv Card Electrophysiol 2015;43:151-9.

12. Debruyne P, Rossenbacker T, Collienne C, et al. Unifocal Right-Sided Ablation Treatment for Neurally Mediated Syncope and Functional Sinus Node Dysfunction Under Computed Tomographic Guidance. Circ Arrhythm Electrophysiol 2018;11:e006604.

13. Rivarola EW, Hachul D, Wu T, et al. Targets and End Points in Cardiac Autonomic Denervation Procedures. Circ Arrhythm Electrophysiol 2017;10:e004638. 